\title{
The Pregnant Surgeon
}

\author{
Cristina R. Harnsberger, MD ${ }^{1}$ Jennifer S. Davids, MD, FACS, FASCRS ${ }^{1}$ \\ ${ }^{1}$ Division of Colon and Rectal Surgery, University of Massachusetts \\ Memorial Medical Center, Worcester, Massachusetts \\ Address for correspondence Jennifer S. Davids, MD, FACS, FASCRS, \\ Division of Colon and Rectal Surgery, University of Massachusetts \\ Clin Colon Rectal Surg 2019;32:450-456. \\ Memorial Medical Center, 67 Belmont Street, Worcester, MA 01605 \\ (e-mail: Jennifer.Davids@umassmemorial.org).
}

\begin{abstract}
Keywords

- pregnant surgeon

- breastfeeding surgeon

- maternity leave

- parental leave

The proportion of women entering medicine and surgery is increasing steadily. As more women surgeons have children during residency and practice, it is essential that we adopt a team approach to the pregnant surgeon, as a pregnancy undoubtedly impacts the team and not just the individual. Coauthored by a practicing surgeon mother of 2 (J. D.) and a postgraduate year 8 colorectal surgery fellow mother of 2 who is currently pregnant (C. H.), we aim to review the data while providing some practical advice for pregnant surgeons and those considering pregnancy, as well as their valued colleagues, mentors, and administrators. Physical challenges are discussed by trimester, as are the risks facing the pregnant surgeon, the logistics of transitioning in and out of maternity leave, and postpartum considerations.
\end{abstract}

The proportion of women in medicine has increased dramatically. While in 1965 only $6.9 \%$ of U.S. medical school graduates were women, most recent data from 2017 demonstrate that half of the matriculants are females (50.7\%). ${ }^{1}$ General surgery is still male predominant; $36.6 \%$ of applicants were women in 2017 , and ultimately women comprise $23 \%$ of fulltime faculty positions. ${ }^{2}$ The rigorous nature of the job and negative attitudes toward pregnancy may deter some students from a surgical career. ${ }^{3}$ Despite this, we feel that the passion and commitment to a career in surgery and the desire to start and raise a family are certainly not mutually exclusive.

While in the past a pregnant surgeon rarely existed, you will most certainly encounter one in contemporary practice. Whether you are the pregnant surgeon, it is your colleague or mentee, or you are in a position of leadership, you will be affected. Pregnant surgeons are no longer an insignificant part of the surgical workforce, and this is the new reality. What has been called a "surgical residency baby boom" occurred after 2000 , as $35 \%$ of female residents and $41 \%$ of male residents had a child during residency, compared with 7 and $24 \%$, respectively. ${ }^{4}$ Therefore, it is imperative that we adopt a team approach to the pregnant surgeon. Considering pregnancy a "problem," inconvenience, imposition, or burden to others is counterproductive.

This study addresses the physical, logistical, and financial challenges facing the pregnant surgeon while also providing some practical advice for both pregnant surgeons and those considering pregnancy, as well as their valued colleagues, mentors, and administrators. There is a paucity of data on many of the issues surrounding the pregnant surgeon, and thus we also provide our own perspectives.

\section{Physical Challenges}

There are physical challenges for the pregnant surgeon that vary by trimester. Notably, every pregnancy is different and it is remarkably hard to "plan" and anticipate how you will feel. As such, there is a balance of planning and flexibility to accommodate the unexpected. While the decision to share pregnancy news with one's workplace is highly personal, it is advantageous to be forthcoming with the news not only if symptomatic or in need of workplace accommodations but also to allow for advance planning of the call schedule and maternity leave as much as possible.

\section{First Trimester}

The increase in estrogen and the $\beta$-subunit of human chorionic gonadotropin by the placenta, starting as early as 10 days after fertilization, can cause nausea and vomiting after only 1 month. During the first trimester, nausea and vomiting is reported by $75 \%$, with $35 \%$ requiring therapy.
Issue Theme Surgeon Health; Guest Editor: Jennifer S. Davids, MD, FACS, FASCRS
Copyright $\odot 2020$ by Thieme Medical Publishers, Inc., 333 Seventh Avenue, New York, NY 10001, USA. Tel: +1(212) 584-4662.
DOI https://doi.org/ 10.1055/s-0039-1693012. ISSN 1531-0043. 
Fatigue during early pregnancy due to elevated levels of progesterone can be debilitating. The advice given at prenatal visits to rest often, eat well, and have small meals every 1 to 2 hours, and exercise can be a near impossibility for a surgeon either in training or practice.

Some practical advice is to keep crackers and snacks in your pockets. The post anesthesia care unit (PACU) usually has a supply in case of emergency. Many find some relief from motion sickness bands (which you cannot wear in the operating room [OR] of course), but medication may be necessary for some. The American College of Obstetricians and Gynecologists recommends the use of pyridoxine (vitamin B6) alone or in combination with doxylamine as the first-line therapy for nausea and vomiting in pregnancy. ${ }^{5}$ Doxylamine can cause drowsiness and is used alone as a sleep aid; therefore, we suggest not taking this prior to working. Evidence is limited on the safety of serotonin 5-HT3 inhibitors such as ondansetron, although its use for pregnancy-related nausea and vomiting appears to be increasing. ${ }^{5}$ If OR smells are bothersome, a drop of peppermint oil on the outside of your mask can make an otherwise nauseating situation tolerable. In anticipation of fatigue during the first trimester, if overnight calls can be minimized, it may be advantageous to do so. Do not overextend workplace or other obligations during this time to allow for sufficient weekend rest after tough weeks in the OR.

\section{Second Trimester}

As pregnancy progresses, many experience relief of nausea and improvement of fatigue; however, many do not. By the second trimester, cardiac output and glomerular filtration rate increase approximately 30 to $50 \%$. As such, urination occurs more frequently and may interrupt a long case. The increase in gastroesophageal reflux can be attributed to elevated progesterone, which relaxes the smooth muscle of the lower esophageal sphincter and diaphragmatic hiatus. Many will need antacids. It is in the second trimester that the gestational diabetes screen is performed, identifying those at risk for hyper- and hypoglycemia. Round ligament pain can cause sharp lower abdominal pain, which may become more common during the second trimester as the enlarged uterus moves around with changes in position. Additionally, leg cramps can occur, which may be related to low iron or potassium.

As the second trimester is usually a good time to operate on a pregnant patient, it is also a relatively "good" time to be a pregnant surgeon. In general, many feel better during the second trimester, and this can be an opportunity to take more calls or book more involved cases. For longer cases, stay well hydrated and plan bathroom breaks at more opportune times such as after the specimen is out, following rigid proctoscopy (before scrubbing back in), or before closing. To minimize reflux, eating small and frequent low-fat meals, and avoiding citrus food, chocolates, and spice can help. For those with gestational diabetes, eating small and frequent meals is also beneficial. Stretching and heating pads can aid in minimizing round ligament pain, as would bananas, meat, or supplements for leg cramps.

\section{Third Trimester}

The third trimester brings its own challenges. Edema is related to an increase in corticotropin-releasing hormone, which ultimately increases aldosterone and cortisol. During the third trimester, compression of the inferior vena cava by the enlarged uterus will exacerbate edema. Back pain is problematic even for the surgeon who is not pregnant. In the third trimester, altered center of gravity, combined with weight gain, can exacerbate or lead to back pain, which is reported in more than two-thirds of pregnant women and typically increases with advancing pregnancy. A Cochrane review of randomized controlled trials found evidence that exercise and osteomanipulative therapy or a multimodal approach (manual therapy, exercise, and education) reduced lower back pain. ${ }^{6}$ Additionally, difficulty sleeping can plague the pregnant surgeon during the third trimester.

Braxton Hicks contractions (false labor contractions) are most commonly noticed in the third trimester and are uterine contractions that do not result in measurable cervical change until they intensify near the onset of true labor. These contractions are unpredictable with irregular intensity, can last as long as 2 minutes, and are typically uncomfortable rather than painful. ${ }^{7}$ Staying hydrated and intermittently changing position can help prevent and limit the frequency of these distracting but harmless contractions during long days in the OR. The advice for alleviation of Braxton Hicks contractions is largely impractical for incorporation into the day of the pregnant surgeon (lie down if you have been standing, take a warm bath, etc.), but having a few extra glasses of water can help as it is thought they may be brought on by dehydration. ${ }^{8}$

Carpal tunnel syndrome can present during pregnancy and can be particularly troublesome for a surgeon. A prospective study of 639 Dutch pregnant women found that carpal tunnel syndrome was reported by $34 \%$, with more severe symptoms after 32 weeks' gestation and independently associated with higher levels of fluid retention and sleeping problems. ${ }^{9}$ Avoiding exacerbating activities, holding surgical instruments in a more ergonomic fashion, taking frequent breaks and performing stretching exercises, and using a heating pad or splint can be helpful to reduce symptoms. Minimizing endoscopy and laparoscopy at the earliest onset of symptoms may limit exacerbation.

There are many strategies for minimizing the physical challenges of the third trimester. Wearing compression stockings is helpful and may prevent development of unsightly and painful varicosities, but ensure that they are lightweight so that you do not overheat. Staying comfortable in hospital-issued scrubs is a challenge in the third trimester. In a pinch, you can use a hemostat to clamp the pants to the top, preventing the embarrassing drop of the pants while scrubbed (do not forget to include the hemostat in the count). Alternatively, wearing a belly band over scrub pants with an untucked top works as well.

At a minimum, regular stretching and a maternity back support belt may be helpful for musculoskeletal pain. Positioning self-retaining retractor systems to accommodate a 
pregnant belly should not be a problem, as surgeons with a large waist circumference have been operating successfully for decades. To manage overheating, cool the room if the patient is euthermic, use ice packs, use a cool washcloth, or break for a drink of cold water. Your OR nurses will be your biggest allies in keeping you comfortable and are true lifesavers in times of need.

A summary of physical challenges by trimester and associated practical recommendations can be found in - Table 1 .

\section{When to Stop Major Cases}

There are no published guidelines on this subject. Anecdotes abound of pregnant surgeons going into labor while operating or having Foley catheters during long cases. Remaining physically active in the form of 150 minutes of moderate intensity exercise per week is recommended throughout an uncomplicated pregnancy ${ }^{10}$; however, "exercise" does not necessarily translate into the physical rigors of operating. Lifting restrictions are vague, but guidelines still in use by obstetricians include repetitive lifting $<11 \mathrm{~kg}$ as a recommended maximum at 40 weeks' gestational age. ${ }^{10}$ Is this equivalent to holding a St. Mark's retractor in an obese pelvis? We may never know and thus recommend a common-sense approach.

During training, one can continue involvement in major cases for as long as one feels physically up to the challenge, given there is a coresident or fellow who could step in and assist the attending surgeon if necessary. Given the strict case numbers and stringent requirements for completion of residency and fellowship, it is to the advantage of the trainee to remain clinically active in the OR even until the estimated date of confinement as long as so permitted by the obstetrician.

As faculty, the decision of when to stop performing major cases is more difficult, as there are no published guidelines. Full term, defined as 37 weeks' gestational age, seems a logical time to transition to minor cases. At term, it would be wise to have a colleague available should assistance become necessary. The same may apply to seeing patients in the office and taking calls; however, it is less altering to a patient's life should their clinic appointment need to be cancelled on a relatively short notice than their surgery. Lastly, while it can be frustrating to the busy pregnant surgeon to taper her practice in anticipation of maternity leave, for some situations, the timing works out fortuitously. For example, a patient with rectal cancer could undergo

Table 1 Challenges and recommendations by trimester

\begin{tabular}{|c|c|}
\hline Challenges by trimester & Practical recommendations \\
\hline \multicolumn{2}{|l|}{ First } \\
\hline Nausea and vomiting & $\begin{array}{l}\text { Keep snacks in your pockets } \\
\text { Eat frequent and small meals } \\
\text { Wear motion sickness bands } \\
\text { Place peppermint oil on you mask to avoid nauseating odors } \\
\text { Talk to your OB about medication options if symptoms are refractory }\end{array}$ \\
\hline Fatigue & $\begin{array}{l}\text { Minimize overnight calls if possible } \\
\text { Minimize workplace and other obligations } \\
\text { Rest on the weekends }\end{array}$ \\
\hline \multicolumn{2}{|l|}{ Second } \\
\hline Reflux & $\begin{array}{l}\text { Eat small low-fat meals, and avoid citrus food, chocolates, spices, and caffeine } \\
\text { Take antacids or talk to your obstetrician about medications options }\end{array}$ \\
\hline Frequent urination & $\begin{array}{l}\text { Stay well-hydrated despite the inconvenience of doing so } \\
\text { Plan opportune times to take bathroom breaks during long cases }\end{array}$ \\
\hline If you are feeling better & $\begin{array}{l}\text { Schedule longer cases } \\
\text { Make up any missed call }\end{array}$ \\
\hline \multicolumn{2}{|l|}{ Third } \\
\hline Edema & $\begin{array}{l}\text { Wear compression stockings } \\
\text { Wear loose-fitting shoes }\end{array}$ \\
\hline Overheating & $\begin{array}{l}\text { Stay well-hydrated } \\
\text { Ensure socks are lightweight } \\
\text { Decrease the room temperature, use ice packs or a cool washcloth } \\
\text { Take a break and drink a glass of cold water }\end{array}$ \\
\hline Carpal tunnel & $\begin{array}{l}\text { Minimize laparoscopy and endoscopy } \\
\text { Take breaks and stretch wrists frequently } \\
\text { Wear a wrist splint } \\
\text { - Use a heating pad }\end{array}$ \\
\hline Back pain & $\begin{array}{l}\text { Stretch frequently } \\
\text { Use a heating pad } \\
\text { Wear a maternity back support belt }\end{array}$ \\
\hline
\end{tabular}


neoadjuvant chemoradiation during the later portion of the surgeon's pregnancy and be ready for surgery once the pregnant surgeon returns from maternity leave.

\section{Risks for the Pregnant Surgeon}

\section{Pregnancy Risks: Infertility, Advanced Maternal Age, Preterm Labor}

Delaying pregnancy due to career aspirations is commonplace in surgery. A survey of 365 female urologists found that they were 7 to 8 years older than the average American woman at the time of their first child. ${ }^{11}$ Findings were similar in thoracic surgery, as a survey of 113 female thoracic surgeons revealed that $98 \%$ delayed pregnancy. ${ }^{12}$ In 2014 , a study by Phillips et al demonstrated that female surgeons had their first child at a mean age of 33.1 years, which increased to 35.4 years if reproductive assistance was necessary. ${ }^{13}$ On average, surgeons in the United States start their first staff appointment at the age of 36 years. ${ }^{14}$ Advanced maternal age is considered age 35 years or older and is associated with infertility, cesarean delivery, low-birthweight infants, and neonatal intensive care unit (NICU) admission. ${ }^{15}$ Therefore, a planned delay in childbearing until completion of surgical training incurs the aforementioned risks.

Are risks for pregnant women in procedural specialties different from women in other areas of medicine? A study by Scully et al. sought to answer this question. Although women in procedural fields were slightly older than their nonprocedural counterparts, time to conception, rates of reproductive assistance use, missed work due to pregnancyrelated issues, preterm labor, and cesarean section were not statistically different between groups. ${ }^{16}$ Results from the postgraduate trainee cohort revealed similar findings. Specifically, trainees in procedural specialties were slightly older than their nonprocedural colleagues and were more likely to require reproductive assistance, but there were no differences in missed work due to pregnancy-related issues, preterm labor, and cesarean section rate. Notably, this study did not include those who were ultimately unsuccessful in becoming pregnant and thus may underestimate the extent of the issue. ${ }^{17}$

\section{Occupational Hazards}

In general, occupational risks to pregnant surgeons are the same as for the nonpregnant surgeon population (and are discussed in detail in the chapter on Workplace Exposures on page $\mathrm{XXX}$ ), although the magnitude of the exposure risk and treatment may differ. In general, taking the usual appropriate safety measures and invoking universal precautions are the best strategies.

There are, however, a few specific occupational risks for the pregnant surgeon that are worthy of further discussion. Minimizing exposure to anesthetic gasses and avoidance of iodine-based scrub are advised as the former crosses the placenta and can affect the fetus, and the latter can affect development of the thyroid or cause maternal subclinical hypothyroidism.
Standard precautions when handling sharps and use of double gloves should minimize exposure to the hepatitis $C$ virus (HCV) and HIV. In the unfortunate event that the pregnant surgeon is exposed, the risk of seroconversion is approximately $0.3 \%$ for HIV and approximately $2 \%$ for HCV. A qualitative test for HCV RNA should be performed 6 to 8 weeks from exposure and treatment should be initiated immediately if positive. ${ }^{18}$ Maternal to fetal transmission of $\mathrm{HCV}$ is rare and occurs almost exclusively in viremic women, and, similarly, transmission of HIV to the fetus can be reduced to $<1 \%$ with the appropriate management. ${ }^{19}$

In contrast to some other surgical and procedural specialties, exposure to ionizing radiation is generally rare in colon and rectal surgery aside from placement of sacral neuromodulators and endoluminal colonic stents. In such cases, the dose of radiation is well under the threshold for what is considered safe, although it is important to monitor cumulative exposure during pregnancy and to always wear well-fitting lead. Most state guidelines consider exposures under 50 mrem per month to be a safe dose, corresponding to 100 to 1,000 fluoroscopic examination of 5 minutes each. $^{20}$

\section{Maternity Leave}

A staggering number of surgical training programs and institutions do not have an official maternity leave policy. Lack of an established, transparent policy can result in stress on behalf of both the pregnant trainee and program director. It may also dissuade trainees from having the potentially dreaded "conversation" till they are further along in pregnancy, leaving less time to make appropriate coverage arrangements. A study of 54 plastic surgery programs found that only $36.5 \%$ had a formal maternity leave policy, and of those that did, only half included plans for coverage should pregnancy complications arise. ${ }^{21}$ Fewer than half of the programs with maternity leave policies specified call requirements and coresidents' roles for coverage. Of the program directors without a specified maternity leave policy, 25\% thought that doing so would lead to insufficient weeks of training and clinical experience.

Given the lack of formal maternity leave policies in surgical residency programs, it is not surprising that the duration of maternity leave in trainees has been shown to be short, as suggested by the literature. A survey of 738 postgraduate medical trainees found that procedural trainees were significantly more likely than their nonprocedural colleagues to have a short maternity leave (defined as $<6$ weeks after vaginal delivery and $<8$ weeks after cesarean section). ${ }^{17}$ Furthermore, procedural trainees were more likely to rely on themselves or their peers to arrange coverage for missed work, and this translated to lower overall career satisfaction. Similar results were found in a survey of 243 female urologists, as a short maternity leave was twice as common in trainees and correlated with decreased career satisfaction. ${ }^{22}$

With this in mind, the American Board of Surgery (ABS) allows for 6 weeks off per year in either of the last 2 years of residency without prior approval. Similarly, residents may 
take an additional 2 weeks off during the first 3 years of residency (to complete 142 weeks instead of 144) to accommodate medical conditions including pregnancy. ${ }^{23}$ And although it requires prior approval from the ABS, the chief year can be extended through the end of August, or five clinical years of residency can be completed in 6 years.

In accordance with the maternity leave specified by the Family and Medical Leave Act in $1993,{ }^{24}$ the Association of Women Surgeons proposed that women surgeons be allowed 4 weeks of paid maternity leave in addition to a 2-week vacation, additional timeoff not be a reason for termination, and residents would not have to make up for missed calls. ${ }^{25}$ These were by no means binding requirements, and it was left up to the discretion of program directors and institutions to formalize a maternity leave policy.

The financial impact of maternity leave is significant. A study by Scully et al reported that female proceduralists contributed a larger percentage of their total household income than their nonproceduralist colleagues. ${ }^{26}$ This study found that 77 to $82 \%$ of female physicians reported losing income due to maternity leave, and $53 \%$ of women in procedural fields reported losing greater than US\$10,000. The vast majority of female proceduralists had a contract that did not include maternity leave, and more than half (54\%) of those who did attempt to negotiate for leave were unsuccessful. Upon return to work, $23 \%$ of proceduralists had to complete missed call shifts, $4.9 \%$ owed money to their practice, and $20 \%$ lost a productivity bonus as many had compensation plans based on relative value unit (RVU) targets. $^{26}$ These findings beg for a better solution. Ideally, one would negotiate maternity leave into her contract, with stipulations for duration of leave, protection from excess calls, financial compensation, and adjustment of RVU targets. Furthermore, while it is beyond the scope of this study, we would also like to stress the importance of incorporating a fair and transparent parental leave policy, as the transition to parenthood does not solely impact the mother.

Lastly, it is important to set realistic expectations for work productivity during maternity leave. Far from vacation, maternity leave is exceedingly exhausting emotionally and physically. It is also a critical bonding period between the mother and the baby. Do not plan to use this time to "catch up" on missed work, attend research meetings, or complete projects.

\section{Postpartum Challenges}

Returning to practice following maternity leave is challenging-in many ways much more so than pregnancy. Mornings become unpredictable as there is now a baby to feed and clothe, and items to prepare for whoever will be caring for the child. Many mornings come in the wake of sleepless nights. As such, arriving to work on time can be stressful and will usually involve a team effort. Some may elect to start their workday later if this is logistically possible within the confines of the busy practice setting. Additionally, there are an abundance of well-baby pediatrician visits, which most mothers would like to attend if possible. Other significant challenges include prolonged NICU stays, neonatal illness, and younger siblings at home. Caring for a baby is a team effort, and, often, roles of both parents evolve in a shared parenting and earning framework. ${ }^{27}$

Plan for more help with childcare and household tasks than you think you may need, as no one has ever complained about having "too much help." Help with childcare is obvious, but also making arrangements for house cleaning, errands, laundry, and other time-consuming housework can be critical. Chronic sleep deprivation makes the postpartum period challenging as well. Consider a graduated increase in work responsibilities or returning on a part-time basis. This is challenging, as the covering partners have been eagerly awaiting your return. For administrators and department chairs, recognition of these significant postpartum challenges and having flexibility during this time of transition are essential to the long-term success of the surgeon mother.

\section{Childcare}

Finding childcare can be quite stressful. For those of us who do not have family or a stay-at-home partner immediately able to provide childcare, finding a nanny, au pair, or day care is time-consuming. Even the most resilient stay-at-home partners need a break, especially considering the long hours they will be with the child while the surgeon is at work. Start the childcare search well in advance of the anticipated delivery, particularly if in search of a day care. Many facilities have long waitlists, as people place their unborn child on the waiting list the moment they find out they are pregnant. Often hospitals have affiliated day cares, with somewhat more flexible and extended hours, although most surgeons' hours will require help with pickups and/or drop-offs. Your hospital may also have an e-mail or webbased nanny Listserv, which may provide recommended nannies from other physicians whose needs may have changed. Advantages to a nanny or au pair are flexibility and care for the baby even during sickness. Some even find two nannies, as the full-time hours of a single employee may not suffice, particularly in a dual-career family. Despite the challenges, finding a fantastic childcare solution is possible. Speaking from our personal experiences, a wonderful caregiver can become loved as part of the family.

\section{Breastfeeding}

Accommodating breastfeeding during return to practice is often the most difficult postpartum challenge. The American Academy of Pediatrics recommends exclusive breastfeeding for approximately 6 months, with continuation for 1 year or longer if mutually desired by the mother and the infant. ${ }^{28}$ Similarly, the World Health Organization (WHO) recommends exclusive breastfeeding for at least 6 months. ${ }^{29}$ Additionally, in some settings, there are cultural expectations and stigma associated with formula feeding of newborns. As a result, mothers can experience a significant amount of pressure from the society and their physicians regarding breastfeeding. However, initiating and sustaining lactation 
remains a challenge for many women. ${ }^{28}$ A survey-based study of mothers who are physician demonstrated that of those who initiate breastfeeding, $41.7 \%$ sustain to 1 year, which is better than the national rate of $27 \%$. However, less than one-third reported the ability to sustain breastfeeding to their personal goal. The most frequently cited challenges included inadequate time, schedule inflexibility, and insufficient space. ${ }^{30}$

At the point of returning to work, the formerly pregnant surgeon is getting even less sleep than previously, managing the emotions and concerns involved with leaving a new baby at home, and has caloric requirements higher than during pregnancy ( 500 vs. 300 extra kCal/day). Feeding a newborn generally occurs every 3 hours during the day, and therefore pumping breast milk should occur at roughly the same frequency to avoid diminishing supply. Accordingly, an appropriate place to do so with access to an electrical wall outlet and a refrigerator is necessary. Unfortunately, designated lactation rooms are often located far from the ORs in a hospital, if there are actually any at all.

Among plastic surgery training programs, only $20 \%$ of those surveyed who had formal maternity leave policies also had defined allowances for breastfeeding upon return to work. ${ }^{21}$ It requires dedication, openness, and flexibility of the entire care team to facilitate regular pumping breaks during the OR for a surgical trainee, but it is possible if desired.

Staff surgeon mothers who continue to breastfeed upon returning to work have their own set of challenges. While they may be able to arrange their schedule to accommodate pumping, a day in the life of a surgeon is far from predictable. The greatest challenge is with long OR cases, whether expected or unplanned. Some practical advice to maximize your time operating is to pump immediately before a case, right up until the time of incision. Have an assistant facilitate the setup. Keep a pump in a locker or nearby area to minimize setup and bring icepacks if a refrigerator is not readily available. A capable trainee may be able to proceed independently in a limited fashion. Even a quick break can help prevent leaking until a more opportune time is available. Ultimately, while there are no perfect solutions for the lactating surgeon, choosing to entirely forgo long cases during breastfeeding may be an even greater challenge to the early career surgeon, often at the cost productivity and potentially lost referrals.

Ultimately, it is a personal choice if or how long to breastfeed based on what is best for the mother and child. Formula feeding is not a failure. A study of exclusively formula-fed infants using age-based formulas from $\leq 14$ days through 4 months demonstrated noninferiority for growth metrics when compared with WHO standards. ${ }^{31}$ There are multiple strategies for low milk supply, including pumping more frequently, increasing rest, fluid intake, and adding various foods such as oatmeal, almonds, or fenugreek seeds. By contrast, if desired, to facilitate a gradual wean from breastfeeding, certain herbs (parsley, peppermint, sage, oregano), topical application of cabbage leaves, antihistamines, and gradually eliminating pumping can accelerate the process while minimizing discomfort associated with engorgement.

\section{Conclusion}

As more women join the surgical workforce, pregnant surgeons are becoming increasingly common. There are significant challenges faced by the pregnant and postpartum surgeon, as well as her colleagues and administrators, but with awareness, planning, and a supportive environment, there are sustainable solutions. By raising awareness of the specific physical, logistical, and financial challenges facing the pregnant surgeon, we hope to prepare pregnant surgeons, their colleagues, mentors, and administrators. The commitment to a healthy and sustainable pregnancy and maternity leave for surgeons is a worthy investment to sustain a full and productive career thereafter.

Conflict of Interest

None declared.

\section{References}

1 US Medical School Applications and Matriculants by School. State of Legal Residence, and Sex, 2016-2017. AAMC.org. Available at: https://www.aamc.org/data/facts/applicantmatriculant/. Accessed February, 2018

2 US Medical School Applications and Matriculants by School. State of Legal Residence, and Sex, 2016-2017. AAMC.org. Available at: https://www.aamc.org/data/facts/. Accessed February, 2018

3 Park J, Minor S, Taylor RA, Vikis E, Poenaru D. Why are women deterred from general surgery training? Am J Surg 2005;190(01): 141-146

4 Frangou C The Expecting Surgeon. General Surgery News. 2017 Mar;44(3). New York, NY: McMahon Publishing

5 Committee on Practice Bulletins-Obstetrics. ACOG Practice Bulletin No. 189: nausea and vomiting of pregnancy. Obstet Gynecol 2018; 131(01):e15-e30

6 Liddle SD, Pennick V. Interventions for preventing and treating low-back and pelvic pain during pregnancy. Cochrane Database Syst Rev 2015;(09):CD001139

7 Raines DA, Whitten RA. Braxton Hicks Contractions. Treasure Island, FL: StatPearls; 2018

8 American College of Obstetricians and Gynecologists. Physical Activity and Exercise During Pregnancy and the Postpartum Period. Available at: https://www.acog.org/Clinical-Guidanceand-Publications/Committee-Opinions/Committee-on-Obstetric -Practice/Physical-Activity-and-Exercise-During-Pregnancy-and -the-Postpartum-Period. Accessed February, 2018

9 Meems M, Truijens S, Spek V, Visser LH, Pop VJ. Prevalence, course and determinants of carpal tunnel syndrome symptoms during pregnancy: a prospective study. BJOG 2015;122(08):1112-1118

10 Council on Scientific Affairs. Effects of pregnancy on work performance. JAMA 1984;251(15):1995-1997

11 Lerner LB, Stolzmann KL, Gulla VD. Birth trends and pregnancy complications among women urologists. J Am Coll Surg 2009;208 (02):293-297

12 Pham DT, Stephens EH, Antonoff MB, et al. Birth trends and factors affecting childbearing among thoracic surgeons. Ann Thorac Surg 2014;98(03):890-895

13 Phillips EA, Nimeh T, Braga J, Lerner LB. Does a surgical career affect a woman's childbearing and fertility? A report on pregnancy and fertility trends among female surgeons. J Am Coll Surg 2014;219(05):944-950

14 Jeekel J. Crucial times for general surgery. Ann Surg 1999;230(06): 739-741

15 Berkowitz GS, Skovron ML, Lapinski RH, Berkowitz RL. Delayed childbearing and the outcome of pregnancy. N Engl J Med 1990; 322(10):659e664 
16 Scully RE, Stagg AR, Melnitchouk N, Davids JS. Pregnancy outcomes in female physicians in procedural versus non-procedural specialties. Am J Surg 2017;214(04):599-603

17 Davids JS, Scully RE, Melnitchouk N. Impact of procedural training on pregnancy outcomes and career satisfaction in female postgraduate medical trainees in the United States. J Am Coll Surg 2017;225(03):411-418.e2

18 Fisher WD. Hepatitis C and the surgeon. Can J Surg 2013;56(02): 80-81

19 Yee LM, McGregor DV, Sutton SH, Garcia PM, Miller ES. Association between maternal HIV disclosure and risk factors for perinatal transmission. J Perinatol 2018;38(06):639-644

20 Shaw PM, Vouyouka A, Reed A. Time for radiation safety program guidelines for pregnant trainees and vascular surgeons. J Vasc Surg 2012;55(03):862-868.e2

21 Garza RM, Weston JS, Furnas HJ. Pregnancy and the plastic surgery resident. Plast Reconstr Surg 2017;139(01):245-252

22 Lerner LB, Baltrushes RJ, Stolzmann KL, Garshick E. Satisfaction of women urologists with maternity leave and childbirth timing. J Urol 2010;183(01):282-286

23 The American Board of Surgery Leave Policy. Available at: http:// www.absurgery.org/default.jsp?policygsleave. Accessed February, 2018
24 U.S. Department of Labor. Wage and hour division: family and medical leave act. Available at: https://www.dol.gov/whd/fmla. Accessed February, 2018

25 Association of Women Surgeons. AWS maternity leave policy for surgical residents. Available at: https://cdn.ymaws.com/womensurgeons.site-ym.com/resource/resmgr/docs/Resource_Library/ ResidentPolicy.pdf. Accessed February, 2018

26 Scully RE, Davids JS, Melnitchouk N. Impact of procedural specialty on maternity leave and career satisfaction among female physicians. Ann Surg 2017;266(02):210-217

27 Sandberg S. Lean. In: Women, Work and the Will to Lead. New York, NY: Alfred A. Knopf; 2013

28 Eidelman AI, Schanler RJ; Section on Breastfeeding. Breastfeeding and the use of human milk. Pediatrics 2012;129(03):e827-e841

29 World Health Organization. Available at: http://www.who.int/ nutrition/topics/exclusive_breastfeeding/en/. Accessed March, 2018

30 Melnitchouk N, Scully RE, Davids JS. Barriers to breastfeeding for US physicians who are mothers. JAMA Intern Med 2018;178(08): 1130-1132

31 Spalinger J, Nydegger A, Belli D, et al. Growth of infants fed formula with evolving nutrition composition: a single-arm non-inferiority study. Nutrients 2017;9(03):E219 